Chapter C-I-5

\title{
Orthogonal Waveforms for High Resolution Range-Doppler Target Reflectivity Estimation
}

\author{
Birsen Yazıcl ${ }^{1}$ \\ ${ }^{1}$ Department of Electrical Computer and Systems Engineering \\ Rensselaer Polytechnic Institute \\ Troy, $N Y 12180$ \\ Email: yazici@ecse.rpi.edu
}

\section{Introduction}

In most radar and sonar applications, the environment to be imaged is extended in range and Doppler space. This means that the environment is composed of several discrete scatterers or a large object with continuum of scatterers in range-Doppler space. In extended environment, the received signal is modeled as a weighted average of the time-delayed and scaled version of the transmitted waveform [1]

$$
e(t)=\int_{-\infty}^{\infty} \int_{0}^{\infty} T_{W}(s, \tau) \frac{1}{\sqrt{s}} p\left(\frac{t-\tau}{s}\right) \frac{d s}{s^{2}} d \tau
$$

where $p$ is the transmitted waveform and $T_{W}$ is the wideband reflectivity density function of the environment associated with each time-delayed and scaled version of the transmitted waveform. $\tau$ is called the time-delay and $s$ is called the Doppler-scale factor given by

$$
s=\frac{c-v}{c+v}
$$

where $c$ is the speed of the transmitted waveform in the propagation medium and $v$ is the radial velocity of scatters.

If the transmitted waveform is narrowband, the model in (1) can be approximated as

$$
e(t)=\int_{-\infty}^{\infty} T_{N}(\omega, \tau) p(t-\tau) \mathrm{e}^{\mathrm{i} \omega t} d \tau d \omega
$$

where $T_{N}$ is called the narrowband reflectivity density function of the environment associated with each time-delayed and frequency-shifted version of the transmitted waveform $p$ and $\omega$ is called the Doppler-shift. 
Equation (1) is referred to as the wideband model and is applicable to scenarios involving both wideband and narrowband transmitted waveforms. Equation (3) is referred to as the narrowband model. The narrowband model is sufficient for most radar applications. However, for sonar and ultra-wideband radar, wideband model is needed [2]. In this chapter, we employ the wideband model due to its generality and the underlying mathematical structure which allows alternative image formation methods.

In radar and sonar, the environment to be imaged is composed of two types of scatterers: Those that are of interests to a practitioner, called target; and those scatterers that are not of interest, called clutter. The objective in radar (sonar) imaging is to estimate the target reflectivity function embedded in clutter and additive thermal noise. Thus, in practice, measurements are modeled as:

$$
y(t)=e_{T}(t)+e_{C}(t)+n(t), \quad t \in\left[t_{0}, t_{1}\right]
$$

where $n$ denotes additive thermal noise, $e_{T}$ and $e_{C}$ denote the received signal due to target $T$ and clutter $C$, respectively. $e_{C}$ is modeled as:

$$
e_{C}(t)=\int_{-\infty}^{\infty} \int_{0}^{\infty} C(s, t) \frac{1}{\sqrt{s}} p\left(\frac{t-\tau}{s}\right) \frac{d s}{s} d \tau .
$$

Figure 1.1 displays the components of the radar (sonar) range-Doppler measurement model.

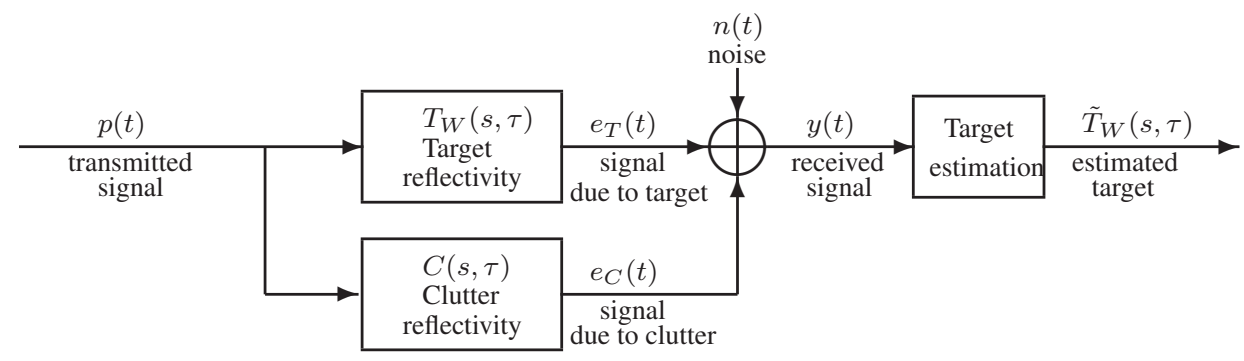

Figure 1.1. A block diagram of the radar range-Doppler measurement model

In this chapter, we address the following two problems: (i) How can we reconstruct the wideband target reflectivity density function embedded in clutter? (ii) What waveforms can we transmit so that the signal due to clutter is "minimized" in some sense?

The reconstruction of wideband target reflectivity was studied before. (See, [3-9] and references therein.) In [7] and [3], the Fourier theory of the affine group was used to develop a method to reconstruct the target reflectivity density function in a 
deterministic setting. In [6], the wavelet transform was used for the reconstruction of the wideband target reflectivity function. However, this approach requires target reflectivity function to be in the reproducing kernel Hilbert space of the transmitted wavelet waveform. In [8,9], the approach in [6] was extended to include affine frames. In all of these studies, the measurements are assumed to be free of additive noise and clutter.

In this paper, we assume that the target to be imaged is embedded in clutter and developed an statistical approach that addresses the target estimation and waveform design problems jointly. We show that with the transmission of appropriate waveforms, the received signal due to clutter can be minimized and a high resolution reconstruction of the target in range-Doppler space can be achieved. Our approach results in the following advantages: (i) Efficient use of transmitted waveform power. For a fixed transmitted signal power, our approach results in higher signal-to-clutter-ratio (SCR) in received measurements. (ii) Reduced computational complexity in receive processing. Clutter suppression is performed via transmission of appropriate waveforms as opposed to processing in receive. (iii) High range-Doppler resolution imaging. The affine Fourier theory based image formation results in an ideal point spread function (PSF), i.e., Dirac-delta function in range and Doppler space, as opposed to the wideband ambiguity function which results from matched-filtering based approach.

Our approach is based on the observation that the received signal can be treated as the affine group Fourier transform of the wideband reflectivity density function evaluated at the transmitted waveform. We design a Wiener filter in the affine group Fourier transform domain as presented in [10] to suppress clutter. We then modify the Wiener filter with an operator associated with the affine group Fourier transform and develop a preconditioner for the transmitted waveforms. We show that when the waveforms are transformed by the preconditioner before transmission, the received signal due to clutter is minimized in the mean square error (MSE) sense. Next, we show that, if $N$ waveforms are to be transmitted, the eigenfunctions of the preconditioner corresponding to largest $N$ eigenvalues are the optimal waveforms in the MSE sense.

The chapter is organized as follows: In Section 2, we provide a review of the affine group and its Fourier transform. In Section 3 we present the estimation of target reflectivity function in a statistical setting. In Section 4, we present the derivation of the optimal clutter rejecting waveforms. In Section 5, we demonstrate the performance of our target estimation and waveform design method. Finally, Section 6 concludes our discussion.

Note that in this chapter we limit our discussion to the clutter suppression and design of clutter rejecting waveforms. For the suppression of additive noise, see our work in [11]. 


\section{Fourier Theory Of the Affine Group}

\subsection{Affine Group}

Affine group or the $a x+b$ group is a 2-parameter Lie group whose elements are given by $2 \times 2$ matrices of the form

$$
\left[\begin{array}{ll}
a & b \\
0 & 1
\end{array}\right], \quad a \in \mathbb{R}^{+}, \quad b \in \mathbb{R}
$$

parameterized by the scale parameter $a$ and the translation parameter $b$.

The affine group operation is the usual matrix multiplication, i.e. $(a, b)(c, d)=$ $(a c, a d+b)$ and the inverse elements are given by the matrix inversion $(a, b)^{-1}=$ $\left(a^{-1},-a^{-1} b\right)$. This defines the affine group as a semi-direct product of the additive group $(\mathbb{R},+)$ and the multiplicative group $\left(\mathbb{R}^{+}, \times\right)$. For the rest of the chapter, we shall denote the affine group by $\mathbb{A}$.

Let $(s, \tau) \in \mathbb{A}$, and let $L^{2}\left(\mathbb{A}, s^{-2} d s d \tau\right)$ and $L^{1}\left(\mathbb{A}, s^{-2} d s d \tau\right)$ denote the space of square summable and absolutely summable functions over $\mathbb{A}$, respectively, i.e.

$$
\int_{\mathbb{A}}|f(s, \tau)|^{2} \frac{d s}{s^{2}} d \tau<+\infty, \quad \int_{\mathbb{A}}|f(s, \tau)| \frac{d s}{s^{2}} d \tau<+\infty,
$$

where $s^{-2} d s d \tau$ is the left Haar measure of the affine group. The inner product of two functions $f_{1}$ and $f_{2}$ in $L^{2}\left(\mathbb{A}, s^{-2} d s d \tau\right)$ is defined as

$$
\left\langle f_{1}, f_{2}\right\rangle=\int_{\mathbb{A}} f_{1}(s, \tau) \overline{f_{2}(s, \tau)} \frac{d s}{s^{2}} d \tau
$$

Affine group is a nonunimodular group, where the right Haar measure is $s^{-1} d s d \tau$. Note that for the affine group, the modular function is given by $\Delta(s, \tau)=s^{-1}$.

\subsection{Fourier Transform over the Affine Group}

There are exactly two nonequivalent, infinite dimensional, irreducible, unitary representations of the affine group, i.e. $\lambda \in\{+,-\}, U_{ \pm}(s, \tau)$. Let $U_{+}$act on the representation space $H_{+}$that consists of functions $\varphi_{+}$, whose Fourier transform are supported on the right half-line and $U_{-}$act on $H_{-}$, the orthogonal complement of $H_{+}$,

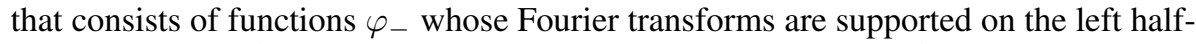
line. Note that $L^{2}(\mathbb{R}, d t)$ is a direct sum of $H_{+}$and $H_{-}$, i.e. $L^{2}(\mathbb{R})=H_{+} \oplus H_{-}$. Then, the representations

$$
U_{ \pm}(s, \tau) \varphi_{ \pm}(t)=\frac{1}{\sqrt{s}} \varphi_{ \pm}\left(\frac{t-\tau}{s}\right)
$$

are unitary, nonequivalent and irreducible in the space $H_{+}$and $H_{-}$, respectively. 
The affine Fourier transform of a function $f \in L^{2}\left(\mathbb{A}, s^{-2} d s d \tau\right)$ is defined as

$$
\mathcal{F}_{ \pm}(f)=\int_{-\infty}^{\infty} \int_{0}^{\infty} s^{-2} d s d \tau f(s, \tau) U_{ \pm}(s, \tau) .
$$

The inverse affine Fourier transform is given by

$$
f(s, \tau)=\sum_{ \pm} \operatorname{trace}\left(U_{ \pm}^{\dagger}(s, \tau) \mathcal{F}_{ \pm}(f) \xi_{ \pm}\right) .
$$

where $U_{ \pm}^{\dagger}(s, \tau)$ denote the adjoint of $U_{ \pm}(s, \tau)$ and $\xi_{ \pm}$are the Hermitian positive definite operators defined as

$$
\xi_{ \pm} \varphi_{ \pm}(t)=\mp \frac{\mathrm{i}}{2 \pi} \frac{d \varphi_{ \pm}}{d t}(t)
$$

The convolution of two functions $f_{1}, f_{2}$ over the affine group is given by

$$
\left(f_{1} * f_{2}\right)(s, \tau)=\int_{-\infty}^{\infty} \int_{0}^{\infty} f_{1}(a, b) f_{2}\left(\frac{s}{a}, \frac{\tau-b}{a}\right) \frac{d a}{a^{2}} d b, \quad(s, \tau) \in \mathbb{A} .
$$

Under the affine group Fourier transform, the convolution of two functions over the affine group becomes operator composition. More specifically,

$$
\mathcal{F}_{ \pm}\left(f_{1} * f_{2}\right)=\mathcal{F}_{ \pm}\left(f_{1}\right) \mathcal{F}_{ \pm}\left(f_{2}\right) .
$$

Let $\left\{s_{ \pm}^{n}(t)\right\}$ denote a set of orthonormal differentiable bases for $H_{ \pm}$, respectively. Define $s^{n}(t)=s_{+}^{n}(t)+s_{-}^{n}(t), U(s, \tau)=U_{+}(s, \tau) \oplus U_{-}(s, \tau),(s, \tau) \in \mathbb{A}$ and $\xi=\xi_{+} \oplus \xi_{-}$. Then for any $p \in L^{2}(\mathbb{R}, d x)$,

$$
U(s, \tau) p=U_{+}(s, \tau) p_{+}+U_{-}(s, \tau) p_{-} .
$$

and if $p$ is differentiable,

$$
\xi p=\xi_{+} p_{+}+\xi_{-} p_{-},
$$

where $p_{+}$and $p_{-}$are orthogonal components of $p$ in $H_{+}$and $H_{-}$, respectively.

For a given orthonormal, differentiable basis $\left\{s_{ \pm}^{n}(t)\right\}$ of $H_{ \pm}$, the inverse affine Fourier transform can be expressed as

$$
\begin{aligned}
f(s, \tau) & =\sum_{ \pm} \sum_{n}\left\langle U_{ \pm}^{\dagger}(s, \tau) \mathcal{F}_{ \pm}(f) \xi_{ \pm} s_{ \pm}^{n}, s_{ \pm}^{n}\right\rangle \\
& =\sum_{n}\left\langle\mathcal{F}(f) \xi s^{n}, U(s, \tau) s^{n}\right\rangle
\end{aligned}
$$

where $\mathcal{F}(f)=\mathcal{F}_{+}(f) \oplus \mathcal{F}_{-}(f)$. 


\section{Target Reflectivity Estimation}

We observe that the wideband received signal model (1) is the affine Fourier transform of the target reflectivity density function $T_{W}$ evaluated at the transmitted waveform $p$, i.e.,

$$
e_{T}(t)=\mathcal{F}\left(T_{W}\right) p(t)
$$

where $e_{T}$ is the received signal due to target. We now assume that the unknown target reflectivity density function, $T_{W}(a, b)$, is a left affine stationary process embedded in additive left affine stationary clutter $C(a, b)$ on the range-Doppler plane. It follows from [10] that the optimal estimator for the target reflectivity density function in the mean square error sense is given by

$$
\tilde{T}_{W}=\left(T_{W}+C\right) * W_{\mathrm{opt}} .
$$

Here, $W_{o p t}$ is the Wiener filter over the affine group given by

$$
\mathcal{F}_{ \pm}\left(W_{\text {opt }}\right)=\left(S_{ \pm}^{T}+S_{ \pm}^{C}\right)^{-1} S_{ \pm}^{T},
$$

where $S_{ \pm}^{T}$ and $S_{ \pm}^{C}$ are the spectral density operators of the target and clutter reflectivity density functions, respectively.

Alternatively, (19) can be expressed as

$$
\mathcal{F}_{ \pm}\left(\tilde{T}_{W}\right)=\mathcal{F}_{ \pm}\left(T_{W}+C\right) \mathcal{F}_{ \pm}\left(W_{\text {opt }}\right)
$$

or

$$
\tilde{T}_{W}(s, \tau)=\sum_{ \pm} \operatorname{trace}\left(U_{ \pm}^{\dagger}(s, \tau) \mathcal{F}_{ \pm}\left(T_{W}+C\right) \mathcal{F}_{ \pm}\left(W_{\mathrm{opt}}\right) \xi\right)
$$

This estimate can be implemented in various forms leading to different adaptive receive and transmit algorithms [11]. Note that both target and clutter spectra, $S_{ \pm}^{T}$ and $S_{ \pm}^{C}$, are not Hermitian operators due to the nonunimodular nature of the affine group. However, it can be shown that $S_{ \pm}^{T} \xi$ and $S_{ \pm}^{C} \xi$ are Hermitian and nonnegative definite. We define

$$
\tilde{S}_{ \pm}^{T}=S_{ \pm}^{T} \xi \quad \text { and } \quad \tilde{S}_{ \pm}^{C}=S_{ \pm}^{C} \xi .
$$

Then, (22) can be rewritten as

$$
\tilde{T}_{W}(s, \tau)=\sum_{ \pm} \operatorname{trace}\left(U_{ \pm}^{\dagger}(s, \tau) \mathcal{F}_{ \pm}\left(T_{W}+C\right) \xi\left(\tilde{S}_{ \pm}^{T}+\tilde{S}_{ \pm}^{C}\right)^{-1} \tilde{S}_{ \pm}^{T}\right) .
$$

Below, we summarize the resulting algorithm to implement the estimate given in (24) and discuss how the estimation problem couples with the waveform design problem. 
Receiver Design - Let $\left\{s_{ \pm}^{n}(t)\right\}$ be a set of orthonormal basis for $H_{ \pm}$, respectively. Then, the target reflectivity estimate in (24) can be expressed as

$$
\begin{aligned}
\tilde{T}_{W}(s, \tau) & =\sum_{ \pm} \sum_{n}\left\langle\mathcal{F}_{ \pm}\left(T_{W}+C\right) \xi\left(\tilde{S}_{ \pm}^{T}+\tilde{S}_{ \pm}^{C}\right)^{-1} \tilde{S}_{ \pm}^{T} s_{ \pm}^{n}, U_{ \pm}(s, \tau) s_{ \pm}^{n}\right\rangle \\
& =\sum_{ \pm} \sum_{n}\left\langle\mathcal{F}_{ \pm}\left(T_{W}+C\right) \tilde{s}_{ \pm}^{n}, U_{ \pm}(s, \tau) s_{ \pm}^{n}\right\rangle
\end{aligned}
$$

where

$$
\tilde{s}_{ \pm}^{n}=\xi\left(\tilde{S}_{ \pm}^{T}+\tilde{S}_{ \pm}^{C}\right)^{-1} \tilde{S}_{ \pm}^{T} s_{ \pm}^{n} .
$$

Note that if $\tilde{s}^{n}=\tilde{s}_{+}^{n}+\tilde{s}_{-}^{n}$ is chosen as the transmitted waveform, then $y^{n}(t)=$ $\mathcal{F}\left(T_{W}+C\right) \tilde{s}^{n}$ becomes the received signal and (25) can be reexpressed as

$$
\tilde{T}_{W}(s, \tau)=\sum_{n}\left\langle y^{n}, U(s, \tau) s^{n}\right\rangle .
$$

This observation leads to the following algorithm for receiver and waveform design:

\section{Algorithm -}

1. Choose a set of orthonormal basis functions $\left\{s_{ \pm}^{n}\right\}$ for $H_{ \pm}$.

2. Transmit $\tilde{s}^{n}=\tilde{s}_{+}^{n}+\tilde{s}_{-}^{n}$, where $\tilde{s}_{ \pm}^{n}=\xi\left(\tilde{S}_{ \pm}^{T}+\tilde{S}_{ \pm}^{C}\right)^{-1} \tilde{S}_{ \pm}^{T} s_{ \pm}^{n}$ for each channel.

3. At the receiver side, perform affine matched-filtering for each channel as follows:

$$
z^{n}(s, \tau)=\left\langle y^{n}, U(s, \tau) \tilde{s}^{n}\right\rangle
$$

where $y^{n}$ is the received signal for the $n$th channel.

4. Coherently sum all channel outputs

$$
\tilde{T}_{W}(s, \tau)=\sum_{n} z^{n}(s, \tau) .
$$

So far, we have not specified how we can choose a set of orthonormal basis functions $\left\{s_{ \pm}^{n}\right\}$ because, the wideband image formation algorithm described above is applicable independent of the choice of transmitted waveforms. The orthogonal functions $\left\{s_{ \pm}^{n}\right\}$ or their filtered counterparts $\left\{\tilde{s}_{ \pm}^{n}\right\}$ do not need to be wideband signals. Thus, this reconstruction formula can be applied to a scenario where there are multiple antenna elements operating independently, each with a limited low resolution aperture (i.e. narrowband transmission). Nevertheless, appropriate processing and fusion of measurements from multiple antenna elements as described above results in a synthetic high resolution image in both range and Doppler. 


\section{Design of Clutter Rejecting Waveforms}

Note that in the target reflectivity estimation outlined above, the minimum mean square error (MMSE) is achieved irrespective of the choice of basis functions or transmitted waveforms. However, the requirement is that a complete set of modified basis functions $\left\{\tilde{s}^{n}\right\}$ must be transmitted to achieve the MMSE. In practice, we are only allowed to transmit a finite number of, say $N$, waveforms. So the question is how to choose the $N$ best waveforms to achieve the MMSE.

We observe that the target reflectivity estimate (22) can be written as

$$
\begin{aligned}
\tilde{T}_{W}(s, \tau) & =\sum_{ \pm} \operatorname{trace}\left(U_{ \pm}^{\dagger}(s, \tau) \mathcal{F}_{ \pm}\left(\tilde{T}_{W}\right) \xi\right) \\
& =\sum_{ \pm} \sum_{n}\left\langle\mathcal{F}_{ \pm}\left(\tilde{T}_{W}\right) \xi s_{ \pm}^{n} U_{ \pm}(s, \tau) s_{ \pm}^{n}\right\rangle
\end{aligned}
$$

where $\left\{s_{ \pm}^{n}\right\}$ are orthonormal bases for $H_{ \pm}, s_{ \pm}^{n}=s_{+}^{n}+s_{-}^{n}$.

Let $T_{n}(s, \tau)=\left\langle\mathcal{F}\left(\tilde{T}_{W}\right) \xi s^{n}, U(s, \tau) s^{n}\right\rangle$. Then,

$$
\tilde{T}_{W}(s, \tau)=\sum_{n} T_{n}(s, \tau) .
$$

It is straightforward to show that $\tilde{T}_{W}(s, \tau)$ and $T_{n}(s, \tau)$ are affine stationary processes with the following properties:

1. $T_{n}(s, \tau)$ and $T_{m}(s, \tau)$ are jointly affine stationary.

2. $E\left[T_{n}(s, \tau) \overline{T_{m}(s, \tau)}\right]=0$ if $n \neq m$.

3. $E\left[T_{n}(s, \tau) \overline{\tilde{T}_{W}(s, \tau)}\right]=E\left[\left|T_{n}(s, \tau)\right|^{2}\right]=\left\langle\mathcal{F}\left(R_{\tilde{T}_{W}}\right) \xi s^{n}, s^{n}\right\rangle$ where $R_{\tilde{T}_{W}}$ is the autocorrelation function of $\tilde{T}_{W}(s, \tau)$.

4. $E\left[\left|\tilde{T}_{W}(s, \tau)\right|^{2}\right]=\sum_{ \pm} \operatorname{trace}\left(\mathcal{F}_{ \pm}\left(R_{\tilde{T}_{W}}\right) \xi\right)$.

5. $R_{\tilde{T}_{W}}=W_{\mathrm{opt}}^{*} *\left(R_{T}+R_{C}\right) * W_{\text {opt }}$, where $W_{\mathrm{opt}}^{*}(s, \tau)=\overline{W_{\mathrm{opt}}\left((s, \tau)^{-1}\right)}$ and $R_{T}$, $R_{C}$ are autocorrelation functions of target reflectivity density process $T(s, \tau)$, and clutter $C(s, \tau)$, respectively.

It follows from the above properties that if only $N$ waveforms are transmitted, then 
the mean square error is given by

$$
\begin{aligned}
E\left[\left|\tilde{T}_{W}-\sum_{n=1}^{N} T_{n}\right|^{2}\right] & =E\left[\left|\tilde{T}_{W}\right|^{2}\right]+\sum_{n=1}^{N} E\left[\left|T_{n}\right|^{2}\right]-2 \sum_{n=1}^{N} E\left[T_{n} \overline{\tilde{T}_{W}}\right] \\
& =\sum_{ \pm} \operatorname{trace}\left(\mathcal{F}_{ \pm}\left(R_{\tilde{T}_{W}}\right) \xi\right)-\sum_{n=1}^{N}\left\langle\mathcal{F}_{\pi}\left(R_{\tilde{T}_{W}}\right) \xi s^{n}, s^{n}\right\rangle \\
& =\sum_{ \pm} \operatorname{trace}\left(\mathcal{F}_{ \pm}\left(R_{\tilde{T}_{W}}\right) \xi\right)-\sum_{ \pm} \sum_{n=1}^{N}\left\langle\mathcal{F}_{ \pm}\left(R_{\tilde{T}_{W}}\right) \xi s_{ \pm}^{n}, s_{ \pm}^{n}\right\rangle \\
& =\sum_{ \pm} \sum_{n=N+1}^{\infty}\left\langle\mathcal{F}_{ \pm}\left(R_{\tilde{T}_{W}}\right) \xi s_{ \pm}^{n}, s_{ \pm}^{n}\right\rangle .
\end{aligned}
$$

Note that

$$
\begin{aligned}
\mathcal{F}_{ \pm}\left(R_{\tilde{T}_{W}}\right) \xi & =\mathcal{F}_{ \pm}\left(W_{\mathrm{opt}}^{*} *\left(R_{T}+R_{N}\right) * W_{\mathrm{opt}}\right) \xi \\
& =\mathcal{F}_{ \pm}\left(W_{\mathrm{opt}}^{*}\right)\left(S_{ \pm}^{T}+S_{ \pm}^{N}\right)\left(S_{ \pm}^{T}+S_{ \pm}^{N}\right)^{-1} S_{ \pm}^{T} \xi=\mathcal{F}_{ \pm}\left(W_{\mathrm{opt}}^{*}\right) \tilde{S}_{ \pm}^{T}
\end{aligned}
$$

Therefore, the MMSE is achieved if $s_{ \pm}^{n}, n=1 \ldots N$, are chosen as the eigenfunctions of the operators $\mathcal{F}_{ \pm}\left(W_{\text {opt }}^{*}\right) \tilde{S}_{ \pm}^{T}$ corresponding to the $N$ largest eigenvalues. Thus, Step 1 of the algorithm introduced in Section 3 can be modified so that the orthonormal functions $\left\{s_{ \pm}^{n}\right\}, n=1, \ldots, N$ are the unit eigenfunctions of $\mathcal{F}_{ \pm}\left(W_{\text {opt }}^{*}\right) \tilde{S}_{ \pm}^{T}$ corresponding to the $N$ largest eigenvalues.

\section{Numerical Experiments}

In this section, we demonstrate the performance of the target estimation and waveform design methods introduced in the previous sections.

For computational convenience, we derived the transmitted waveforms from the Laguerre polynomials.

Let

$$
\hat{s}_{+}^{n}(\omega)=L_{n-1}(\omega) \mathrm{e}^{-\omega / 2}, \quad \omega \in \mathbb{R}^{+}, n \in \mathbb{N},
$$

where $\hat{s}_{+}^{n}(\omega)$ is the Fourier transform of $s_{+}^{n}(t)$ and $L_{n-1}, n \in \mathbb{N}$ are the Laguerre polynomials defined by

$$
\begin{aligned}
L_{0}(x) & =1 \\
L_{1}(x) & =-x+1 \\
L_{n+1}(x) & =\frac{2 n+1-x}{n+1} L_{n}(x)-\frac{n}{n+1} L_{n-1}(x), \quad n \in \mathbb{N} .
\end{aligned}
$$


It is well-known that [12]

$$
\int_{0}^{\infty} \mathrm{e}^{-x} L_{m}(x) L_{n}(x) d x= \begin{cases}1 & m=n \\ 0 & \text { else }\end{cases}
$$

Therefore, $\left\{\hat{s}_{+}^{n}\right\}$ is an orthonormal basis for $L^{2}\left(\mathbb{R}^{+}, d x\right)$. Let $s_{+}^{n}$ be the standard inverse Fourier transform of $\hat{s}_{+}^{n}$. Then, $\left\{s_{+}^{n}\right\}$ is an orthonormal basis for $H_{+}$. Let $\hat{s}_{-}^{n}(\omega)=\hat{s}_{+}^{n}(-\omega), \omega \in \mathbb{R}^{-}$. Then, $s_{-}^{n}(t)=\overline{s_{+}^{n}(t)}, t \in \mathbb{R}$ and $\left\{s_{-}^{n}\right\}$ are orthonormal bases for $H_{-}$.

We generated a set of realizations of the target and the clutter based on the following spectral density operators $\tilde{S}_{ \pm}^{T}$ and $\tilde{S}_{ \pm}^{C}$ with respect to bases $\left\{s_{ \pm}^{n}\right\}$

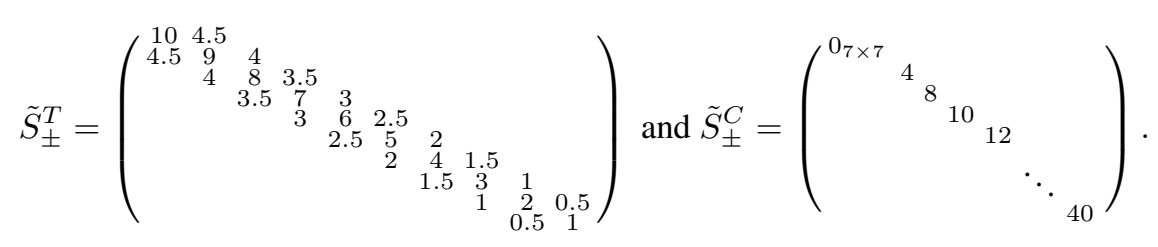

Figures 5.1 (a) and (b) show a realization of the target and target embedded in clutter.

We synthesized 10 realizations of the target and clutter at various signal to clutter ratios (SCR) defined as $S C R=20 \log _{10}\left(\sigma_{s}^{2} / \sigma_{c}^{2}\right)$ where $\sigma_{s}^{2}$ and $\sigma_{c}^{2}$ are the target and clutter reflectivity variance, respectively. We generated each realization according to the spectral density operators $\sigma_{s}^{2} \tilde{S}_{ \pm}^{T}$ and $\sigma_{c}^{2} \tilde{S}_{ \pm}^{C}$.

We chose the transmitted waveforms as

$$
\bar{s}^{n}=\xi\left(\tilde{S}^{T}+\tilde{S}^{C}\right)^{-1} \tilde{S}^{T} s^{n}, \quad n=1, \ldots, 20
$$

Figure 5.1 (c) and (d) show estimated target reflectivity using our method and the method in [7]. Note that the method in [7] uses Hermite polynomial basis. Figure 5.2 shows the mean square error (MSE) between the true and estimated reflectivity density functions for our method and for the method in [7] at different SCR levels. We calculated the MSE by averaging the error over 10 realizations of the true and the estimated target reflectivity at each SCR level. The numerical results show that our method results in a lower MSE than the one in [7], particularly for low levels of SCR.

\section{Conclusion}

We treated the wideband received signal as the affine group Fourier transform of the range-Doppler wideband target reflectivity function evaluated at the transmitted waveform. Our approach provides to a framework in which the high resolution target reflectivity function estimation and waveform design problems are jointly addressed. We developed a Wiener filtering method in the Fourier transform of the affine group to suppress clutter. We, then, showed that Wiener filter can be used to precondition 


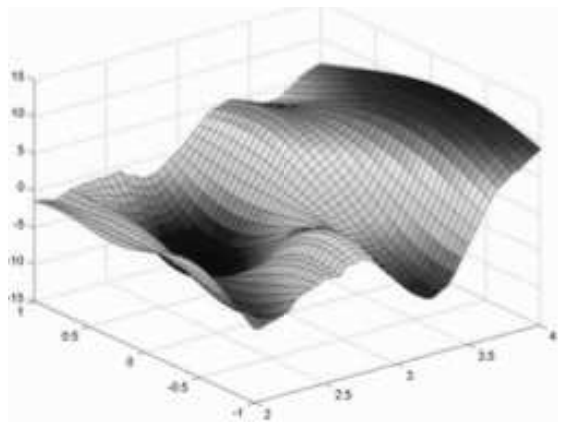

(a) Target

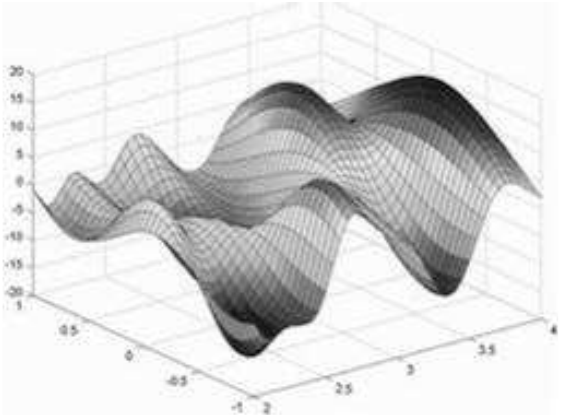

(c) Estimated target by the method in [7]

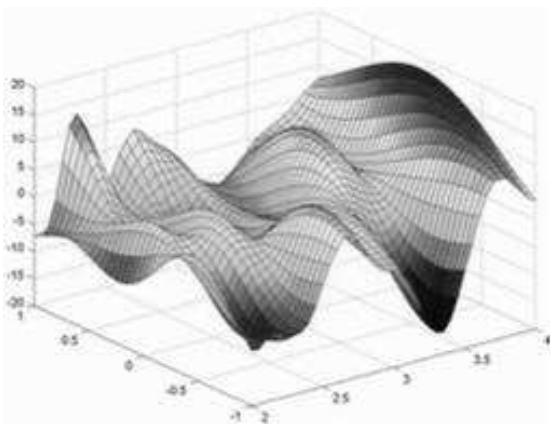

(b) Target embedded in clutter

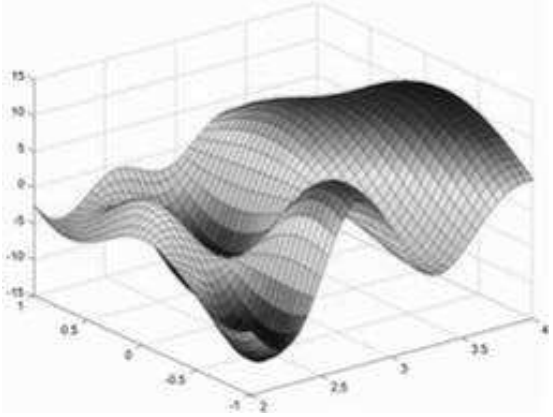

(d) Our method

Figure 5.1. Estimated target reflectivity function embedded in clutter. (a) True target reflectivity function. (b) Target reflectivity function embedded in clutter. (c) Estimated target reflectivity function using the method in [7]. (d) Estimated target reflectivity function by our method.

the transmitted waveforms to reject clutter. When, only $N$ waveforms are to be transmitted, the optimal waveforms in the MMSE sense becomes the eigenfunctions of the modified Wiener filter corresponding to the largest $N$ eigenvalues.

\section{Acknowledgments}

The author is grateful to Air Force Office of Scientific Research (AFOSR) ${ }^{1}$ for supporting this work under the agreements FA9550-04-1-0223, and FA9550-07-1-0363.

\footnotetext{
${ }^{1}$ Consequently the U.S. Government is authorized to reproduce and distribute reprints for Governmental purposes notwithstanding any copyright notation thereon. The views and conclusions contained herein are those of the authors and should not be interpreted as necessarily representing the official policies or endorsements, either expressed or implied, of the Air Force Research Laboratory or the U.S. Government.
} 


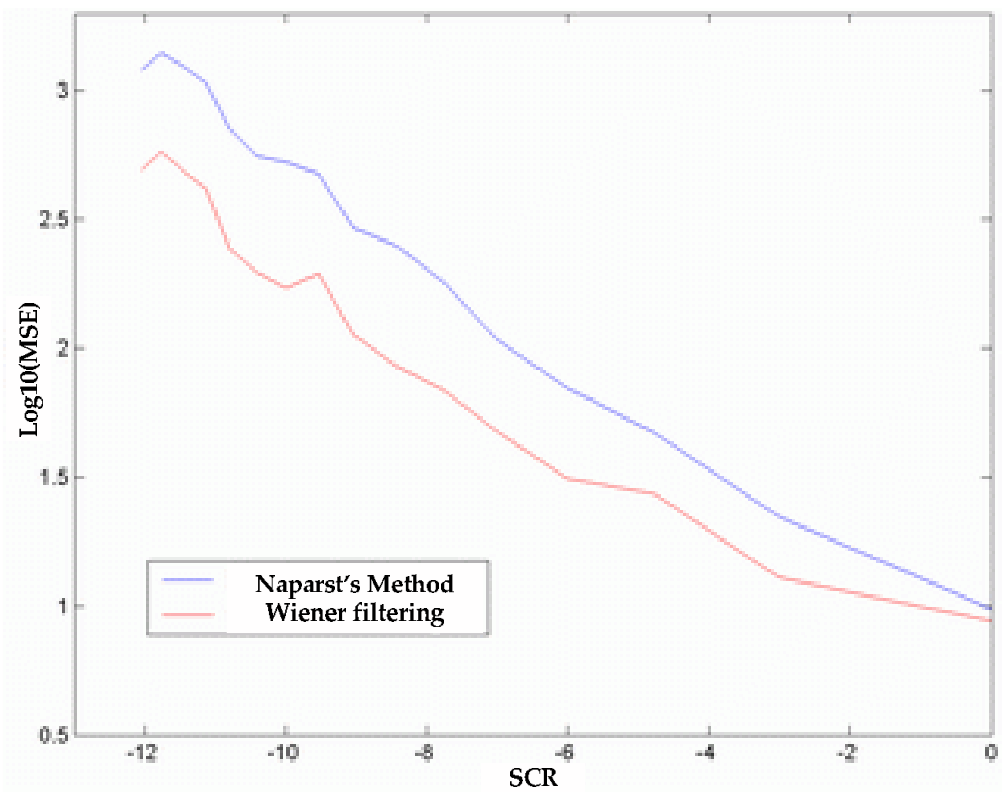

Figure 5.2. Mean square error between the estimated and true target reflectivity function.

\section{References}

[1] R. E. Blahut, Algebraic Methods for Signal Processing and Communications Coding. New York: Springer-Verlag, 1991.

[2] J. Taylor, Introduction to Ultra-wideband Radar Systems. Florida: CRC Press, 1995.

[3] W. Miller, "Topics in harmonic analysis with applications to radar and sonar," in Radar and Sonar Part I, ser. IMA Volumes in Mathematics and its Applications, R. E. Blahut, W. Miller, and C. H. Wilcox, Eds. New York: Springer-Verlag, 1991, pp. 66-168.

[4] C. E. Cook and M. Bernfeld, Radar Signals. New York: Academic, 1967.

[5] D. A. Swick, “A review of wideband ambiguity functions. nrl rep. 6994," Naval Research Laboratory, Washington, DC, Tech. Rep., 1969.

[6] L. G. Weiss, "Wavelets and wideband correlation processing," IEEE Signal Processing Mag., vol. 11, pp. 13-32, 1994.

[7] H. Naparst, "Dense target signal processing," IEEE Trans. Inform. Theory, vol. 37, pp. 317-327, 1991. 
[8] L. Rebollo-Neira, A. Pastino, and J. Fernandez-Rubio, "Frames: a maximum entropy statistical estimate of the inverse problem," Journal of Mathematical Physics, vol. 38, pp. 4863-4871, 1997.

[9] _ - "Reconstruction of the joint time-delay doppler-scale reflectivity density in the wideband regime: A frame theory based approach," Journal of Mathematical Physics, vol. 41, pp. 5325-5341, 2000.

[10] B. Yazıc1, "Stochastic deconvolution over groups," IEEE Transactions on Information Theory, vol. 50, pp. 494-510, March 2004.

[11] B. Yazıcı and G. Xie, "Wideband extended range-dopler imaging and diversity waveform design," IEEE Transactions on Information Theory, vol. 52, pp. 45634580, 2006.

[12] M. Abramowitz and I. A. Stegun, Orthogonal Polynomials, New York, ch. 22. 